\title{
Voiding Dysfunction in Old Male Rats Associated With Enlarged Prostate and Irregular Afferent-Triggered Reflex Responses
}

\author{
Chuan Zhang ${ }^{1,2, *}$, Xuhong Li3 ${ }^{3, *}$, Timothy B. Boone ${ }^{2,4}$, Yolanda Cruz ${ }^{5}$, Yingchun Zhang ${ }^{1,2}$, Alvaro Munoz ${ }^{2,6}$ \\ ${ }^{1}$ Department of Biomedical Engineering, University of Houston, Houston, TX, USA \\ ${ }^{2}$ Regenerative Medicine and Tissue Engineering Program-Urology, Houston Methodist Research Institute, Houston, TX, USA \\ ${ }^{3}$ The Third Xiangya Hospital, Central South University, Changsha, China \\ ${ }^{4}$ Department of Urology, Houston Methodist Hospital, Houston, TX, USA \\ ${ }^{5}$ Centro Tlaxcala de Biología de la Conducta, Universidad Autónoma de Tlaxcala, Tlaxcala, México \\ ${ }^{6}$ Centro Universitario del Norte, Universidad de Guadalajara, Colotlán, Jalisco, México
}

Purpose: This study was conducted to evaluate the hypothesis that an enlarged prostate in old rats may lead to complications associated with voiding dysfunction involving ionotropic P2X2/3-type purinergic receptors

Methods: Intact animals were divided into male young (MYR; 8-10 weeks old) and male old (MOR; 20 months old) rats. The animals underwent simultaneous detrusor electromyography (EMG) and suprapubic cystometry (CMG) under urethane anesthesia. Immunofluorescence techniques were used to evaluate prostatic autonomic innervation and P2X3R expression in bladder urothelial cells. The functional role of P2X3R was characterized by intramuscular application of AF-353, a selective $\mathrm{P} 2 \mathrm{X} 2 / 3 \mathrm{R}$ antagonist.

Results: The prostate index significantly increased in MOR, suggestive of an enlarged prostate affecting micturition patterns. Significant EMG and CMG differences were found between MYR and MOR. Higher immunoreactivity for P2X2/3R in the urothelial layer and for prostatic neurofilaments was seen in MOR. Systemic inhibition of P2X2/3R had minimal effects on MYR responsiveness, but improved voiding function in MOR with a marked decrease of intravesical pressure and bladder contractile responses.

Conclusions: The data support the hypothesis that an enlarged prostate in MOR may contribute to voiding dysfunction involving activation of $\mathrm{P} 2 \mathrm{X} 2 / 3 \mathrm{R}$, which enhances a prostate-bladder reflex. This reflex may increase bladder afferent transmission and activation of increased prostate innervation, leading to voiding dysfunction.

Keywords: Prostate; Urinary bladder; Urothelium; Receptors, Purinergic P2X3; Micturition

- Research Ethics: All experimental procedures were approved by the Institutional Animal Care and Use Committee of Houston Methodist Research Institute (IACUC; ID: AUP-0615-0044/IS00001252; responsible investigator: Alvaro Munoz) and performed in accordance with the Guidelines from the National Institutes of Health on the Care and Use of Laboratory Animals.

- Conflict of Interest: No potential conflict of interest relevant to this article was reported.

\section{- HIGHLIGHTS}

- Immunoexpression of both P2X3 receptors in bladder-urothelium and neurofilaments in prostate tissue from aged male rats is higher than in young animals.

- Experiments performing simultaneous electromyography of the BLADDER DOME with bladder cystometry in anesthetized rats, uncovered clear dysfunctional voiding-patterns in aged male animals, which are improved by systemic inhibition of P2X3 receptors.

- Aging-related changes in the lower urinary tract and prostate from aged male-rats may contribute to voiding dysfunction, involving a hyperexcitable prostate-bladder reflex via activation of urothelial P2X3 receptors.

Corresponding author: Alvaro Munoz (iD https://orcid.org/0000-0002-5726-0810 Centro Universitario del Norte, Universidad de Guadalajara, Carretera Federal No. 23, Km. 191, C.P. 46200, Colotlán, Jalisco, México

E-mail: alvaro.munoz@cunorte.udg.mx

${ }^{\star}$ Chuan Zhang and Xuhong Li contributed equally to this study as co-first authors. Submitted: April 16, 2020 / Accepted after revision: May 24, 2020
This is an Open Access article distributed under the terms of the Creative Commons Attribution Non-Commercial License (https://creativecommons.org/licenses/by-nc/4.0/) which permits unrestricted non-commercial use, distribution, and reproduction in any medium, provided the original work is properly cited. 


\section{INTRODUCTION}

Lower urinary tract symptoms generate elevated financial burdens and healthcare costs in older men [1,2]. One of the most common symptoms is difficulty urinating due to narrowing of the urethral lumen associated with an enlarged prostate, otherwise known as benign prostatic hyperplasia. This condition is not associated with prostate cancer, but generates similar symptoms of bladder outlet obstruction [2].

Although the pathophysiology of voiding dysfunction associated with prostatic hyperplasia is not clearly understood, several pharmacological treatments are available to modestly improve the quality of life in patients with benign prostatic hyperplasia. Common therapies include the use of phosphodiesterase inhibitors, alpha-adrenergic blockers, or 5-alpha reductase inhibitors, but these treatments do not dramatically improve urinary symptoms [3]. Meanwhile, few basic research studies have correlated histological findings with functional evaluations, making it even more difficult to interpret pharmacological effects and targets.

Consequently, more comprehensive preclinical investigations using animal models to evaluate the effects of aging on pathological alterations of the lower urinary tract are necessary. Delineating a mechanistic understanding of voiding dysfunction in the older may be helpful for making clinical diagnoses and selecting better therapeutic interventions.

Several animal models have been used to evaluate bladder outlet obstruction in rats [4]. Some of these involve the mechanically induced partial occlusion of the urethra in rats to simulate similar conditions as those observed in patients with prostate-induced urethral obstructions [5]. Despite the efforts made to evaluate the impact of aging on micturition, the reported results have varied greatly, which may be explained by differences in species, sex, the application of anesthetics, and the experimental procedures used [6].

Thus, to better understand the neurophysiological pathways involved in voiding dysfunction associated with an enlarged prostate, we performed simultaneous detrusor electromyographic and cystometric recordings in aged male rats (20 months old), and compared the responses to those in young animals (8-10 weeks old). This approach was intended to establish naturally occurring benign prostatic hyperplasia in old rats to yield further insights into the origin of the complications associated with voiding dysfunction.

Ionotropic $\mathrm{P} 2 \mathrm{X} 3$ and $\mathrm{P} 2 \mathrm{X} 2 / 3$ purinergic receptors (P2X3R) are hetero- or homo-trimer protein complexes that function as nonselective ion channels activated by adenosine triphosphate (ATP), and participate in afferent transmission of sensory stimuli in different tissues, including the lower urinary tract $[7,8]$. Due to the relevance of these receptors for nociception, various antagonists have been designed for pharmacological use in diseases related to pain, including AF-353, an antagonist able to block $\mathrm{P} 2 \mathrm{X} 3$ and $\mathrm{P} 2 \mathrm{X} 2 / 3$ receptors in the periphery and the central nervous system [9]. Because the bladder sensory symptoms associated with prostate hyperplasia are carried by afferent signals [10], some of them mediated by P2X2/3R [11-13], we pharmacologically tested the role of ionotropic P2X3R [9] in both groups of rats.

\section{MATERIALS AND METHODS}

All experimental procedures were approved by our Institutional Animal Care and Use Committee, and performed in accordance with the Guidelines from the National Institutes of Health on the Care and Use of Laboratory Animals. Rats were housed in a pathogen-free environment, with 12-hour light/ dark cycles, a controlled room temperature of $25^{\circ} \mathrm{C}$, and had ad libitum access to food and water in plastic cages containing corn cob bedding. Efforts were made to minimize the number of animals used, while maintaining the ability to make significant comparisons.

\section{Animals and Surgical Preparation}

Long-Evans rats were divided into groups of male young rats (MYR; 2-2.5 months old; $\mathrm{N}=6$ ) or male old rats (MOR; 20 months old; $\mathrm{N}=6$ ). The MOR animals were donated through the Resources and Tissue-Sharing Initiative at our institution, and at no time participated in any other study. Both MYR and MOR were obtained from a local vendor (ENVIGO, Houston, TX, USA). Animals were weighed and anesthetized with bilateral subcutaneous injections of urethane (Sigma-Aldrich, St. Louis, MO, USA; $1.2 \mathrm{~g} / \mathrm{kg}$ ) at the dorso-thoracic area (half dose per side). After reaching the anesthetic plane, a midline abdominal incision was performed and a suprapubic catheter (PE-50) was sutured onto the bladder dome for intravesical infusion of saline solution and pressure sensing. Additionally, 1 custom-made stainless steel spring electrode (internal diameter $=0.457 \mathrm{~mm}$ ) was placed on the bladder dome $[8,13]$. Abdominal muscles and skin were sutured in 2 layers after surgery. A heating lamp was used to maintain body temperature through- 
out the surgical procedures and experiments.

\section{Cystometry and Electromyography}

The status of both the electrode and suprapubic catheter was verified with a stereomicroscope before placing animals in a prone position. Voided urine was collected with a plastic cup mounted on top of a pressure sensor for monitoring effective volumes. Saline infusion was performed using a syringe pump through the suprapubic catheter, at a rate of $0.1 \mathrm{~mL} / \mathrm{min}$. Before the start of the experiment, continuous saline infusion was performed for approximately 10-15 minutes until stable voiding events, indicated by real-time cystometry (CMG), were observed. After 6 consecutive voiding events, 10 mg/kg AF-353 (diluted at a final dimethyl sulfoxide concentration of $0.01 \%$ in saline solution) was injected intramuscularly into the right quadriceps muscle. This dose was selected based on previous studies in which the P2X3R antagonist was administered intravenously [14]. Based on the biological characterization of AF353 , for which a time to peak plasma concentration of approximately 30 minutes was determined when orally administered [11], we waited for a period of 20 minutes before 6 additional consecutive voiding events were recorded. Electromyography (EMG) signals were amplified with a DP-304 differential amplifier (Warner Instruments, Hamden, CT, USA), acquired at a sampling rate of $2 \mathrm{kHz}$, and digitized using the WinDaq data acquisition software (DataQ Inst. Akron, OH, USA).

\section{Cystometric Analysis}

In contrast to typical cystometric responses, the Long-Evans male rats used in this study showed a far more complicated voiding pattern that involved the presence of nonvoiding contractions (NVCs), small volume voiding events (SVVEs), and effective contractions (ECs) during sustained high intravesical pressures before returning to baseline bladder pressure, particularly in MOR animals (;). Nonetheless, for CMG characterization, only the last 3 voiding events, indicated by visible intraluminal pressure high-frequency oscillations (IPHFOs) at each stage (intact and following intramuscular AF-353 injection) were used. NVCs were considered to occur when a change in bladder pressure higher than $5 \mathrm{~cm} \mathrm{H}_{2} \mathrm{O}$ was observed, accompanied by no evident release of saline, either at baseline or at high intravesical pressures. The frequency was calculated as the number of NVCs over the duration of the recording condition and normalized by minute (i.e., $\mathrm{NVC} / \mathrm{min}$ ). Additionally, the peak pressure during bladder contractions $\left(\mathrm{cm} \mathrm{H}_{2} \mathrm{O}\right)$, intercon- tractile interval (sec), duration of IPHFOs (sec), and voided volume (mL) for ECs were determined using MATLAB R2017a (MathWorks, Natick, MA, USA).

\section{Electromyographic Analysis}

Second-order Butterworth notch filters were applied to remove the power line interference and harmonics for all electrophysiological signals. EMG signals were further bandpass-filtered at 5-400 Hz using a second-order Butterworth filter. A template subtraction method was applied to remove the electrocardiogram contamination. Epochs of bladder EMG discharging were automatically detected using a preset amplitude threshold. The onset/offset of EMG activity was temporally referenced to the bladder pressure peak. The bladder pressure that induced the onset/offset of signal activation was also determined. For each EMG experiment, the signal turns (indicative of frequency), amplitude, and duration were calculated in triplicate, averaged, and grouped according to the age condition.

\section{Morphometric Analysis}

After performing $\mathrm{CO}_{2}$-thoracothomy euthanasia, the ventral, lateral, and dorsal prostate lobes were removed from the MYR and MOR animals and wet-weighed as a whole, and the resulting weight values were recorded. The same procedure was performed for the urinary bladders including tissue from the bladder base to the bladder dome. Thereafter, the bladder and prostate (ventral + lateral + dorsal lobes) indexes were calculated respectively as the ratio of bladder or prostate weight per 100-g body weight.

\section{Immunofluorescent Procedure in Bladder Tissue}

Following systemic treatment with the P2X3R antagonist during simultaneous CMG-EMG evaluations, urinary bladders were processed and stained as previously described [15]. Briefly, equatorial bladder specimens from each animal were incubated in $4 \%$ formalin overnight and cryoprotected in $30 \%$ sucrose at $4^{\circ} \mathrm{C}$. Thereafter, specimens were sectioned at $30 \mu \mathrm{m}$ with a cryostat, and blocked overnight at $4^{\circ} \mathrm{C}(10 \%$ goat serum, $2 \%$ bovine serum albumin, $0.1 \%$ Triton X-100, and $0.05 \%$ Tween 20). After washout, bladder sections were incubated overnight with antibodies against-actin (1:400; AM1829B, ABGENT, San Diego, CA, USA) and P2X3R receptors (1:1,000; AB90905, ABCAM, Cambridge, MA, USA). After washing, sections were incubated in the dark for 2 hours at room temperature with a mixture of donkey anti-rabbit (Alexa Fluor 647; AB150075, 
ABCAM) and goat anti-mouse (Alexa Fluor 488, AB150113, ABCAM) secondary antibodies. Sections were washed, covered with mounting medium containing 4'-6-diamidino-2-phenylindole (DAPI) (ab104139, ABCAM), and protected with glass coverslips.

\section{Immunofluorescent Procedure in Prostatic Tissue}

A section of the ventral prostate lobe was fixed in formalin and then cryoprotected in $30 \%$ sucrose. Briefly, prostatic tissue was sectioned at $30-\mu \mathrm{m}$ thickness and incubated overnight at $4^{\circ} \mathrm{C}$ with a mouse anti-neurofilaments 68 and $200 \mathrm{kDa}$ antibody (1:100; AB1989, Sigma-Aldrich) and $\beta$-actin (1:400). Secondary antibody incubation was performed as described for bladder tissues. Sections were covered with mounting medium containing DAPI and protected with glass coverslips. The antibody solutions were simultaneously used for all bladder and prostate slides.

\section{Immunofluorescent Evaluations}

A dry $\times 40$ objective on a Leica TCS SPE confocal microscope (Leica Microsystems, Buffalo Grove, IL, USA) was used for acquiring images, which were digitized using Leica LAS-X interface software. The image analysis was performed with ImageJ software (U.S. National Institutes of Health, Bethesda, MD, USA) by determining the signal contribution (as percent) for each channel (i.e., DAPI, $\beta$-actin, neurofilaments, and P2X3R) over the full image area. Additionally, the P2X3R signals were normalized to DAPI and $\beta$-actin as immunoreactivity indicators for each bladder.

\section{Statistical Analysis}

Intergroup statistical comparisons between the MOR and MYR groups were performed using 1-way analysis of variance with multiple comparisons between means, and corrected with the Tukey post hoc test. Intragroup analyses were performed with the 2-tailed unpaired $t$-test. All values are expressed as mean \pm standard error. In all cases, the level of statistical significance was set at $\mathrm{P}<0.05$. All statistical analyses were performed with GraphPad 6.0 software (Prism, GraphPad Software Inc., San Diego, CA, USA).

\section{RESULTS}

\section{Morphometric Differences Between MYR and MOR}

As expected, the average body weight of MYR $(289.2 \pm 7 \mathrm{~g})$ was significantly lower than that of MOR $(594.5 \pm 7 \mathrm{~g})(\mathrm{P}<0.001)$.
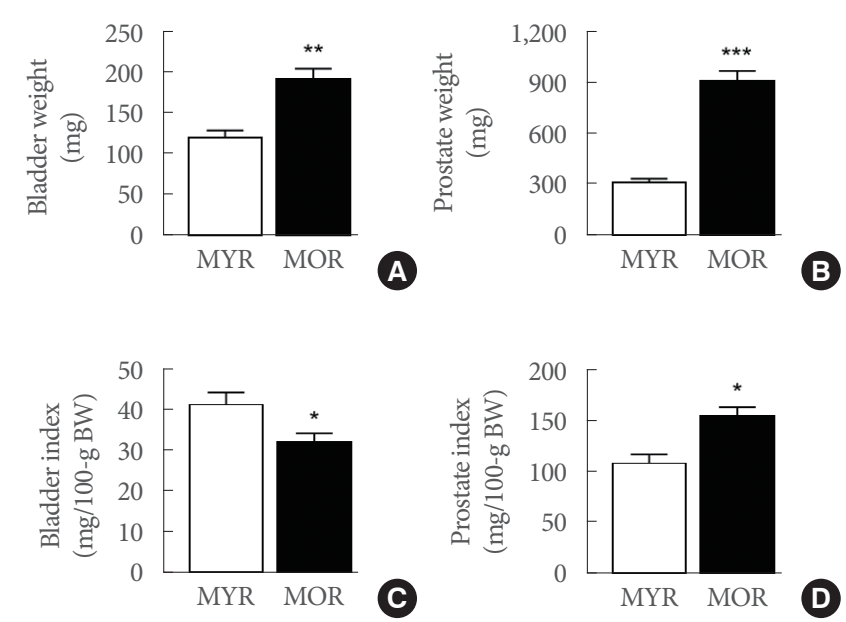

Fig. 1. Morphometric differences between MYR and MOR. Group analysis for bladder weight (A), prostate weight (B), bladder index (C), and prostate index (D) in MYR and MOR animals. ${ }^{*} \mathrm{P}<0.05,{ }^{*} \mathrm{P}<0.01$, and ${ }^{* *} \mathrm{P}<0.001$ vs. MYR group (t-test). MYR, male young rats; MOR, male old rats.

Similarly, the weights of the isolated bladder and prostate tissues were significantly higher in MOR (Fig 1A, B). However, when compared to the MYR animals, a lower bladder index (Fig. 1C) and a higher prostate index were observed in MOR, indicative of an enlarged prostate (Fig. 1D).

\section{Higher Expression of Urothelial P2X3R in MOR}

Both MYR and MOR showed similar levels of DAPI signals (indicative of a comparable numbers of cells), and urothelial immunoreactivity for $\beta$-actin and P2X3R (Fig. 2A-D, MYR; Fig. $2 \mathrm{E}-\mathrm{H}, \mathrm{MOR}$ ). In an analysis of the total contribution of DAPI, $\beta$-actin, and P2X3R to the total signal area, higher values were found for P2X3R in MOR than in MYR (Fig. 2I; P < 0.01). When the P2X3R signal contribution was normalized to either DAPI ( $P<0.05$, MOR vs. MYR) or $\beta$-actin $(\mathrm{P}<0.01$, MOR vs. MYR), a greater index remained for urothelial P2X3R in MOR than in MYR (Fig. 2J).

\section{Higher Expression of Neurofilaments in Ventral Prostate Lobes From MOR}

Immunoreactivity for $\beta$-actin and neurofilaments, as well as DAPI staining, was observed in the prostatic ventral lobes from both MYR (Fig. 3A-D) and MOR (Fig. 3E-H). A group analysis of these 3 markers showed a significantly higher expression of neurofilaments in MOR prostatic tissues (Fig. 3I) $(\mathrm{P}<0.05$ vs. MYR). 

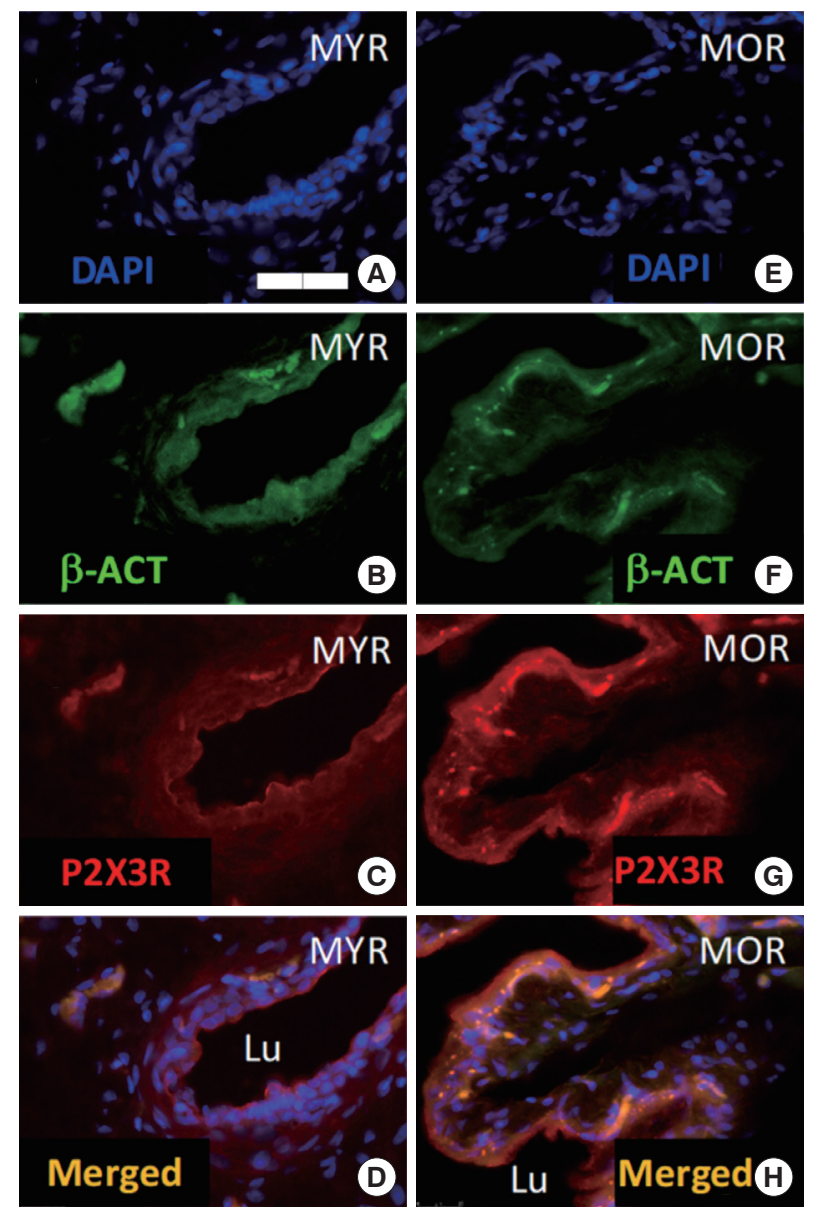
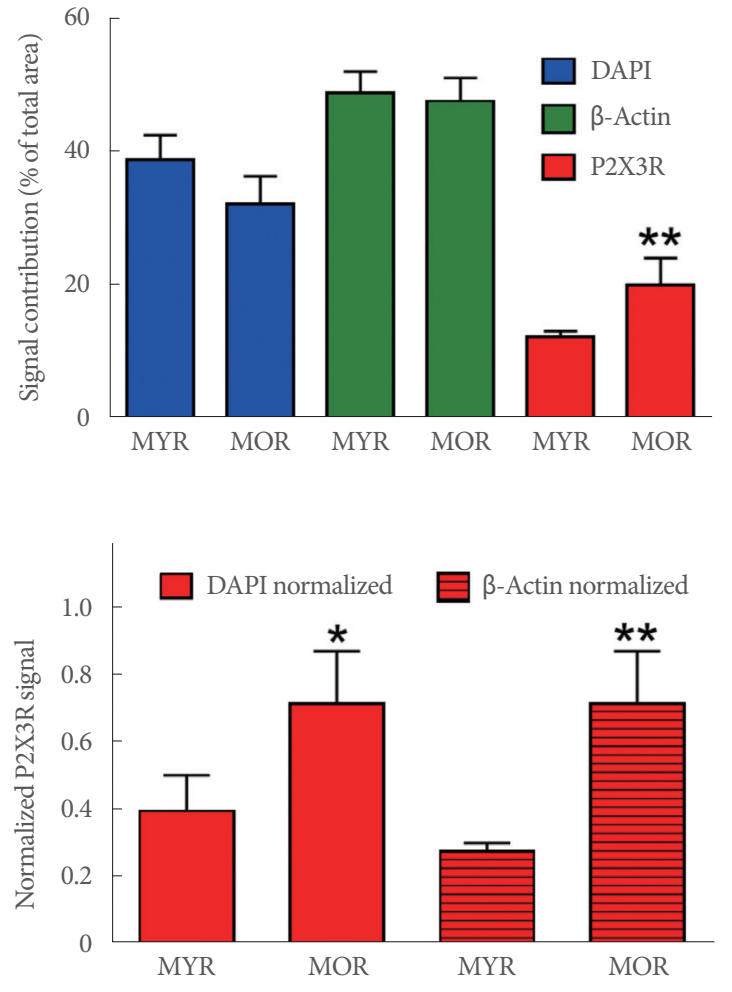

(1)

Fig. 2. The urothelial immunoexpression of $\mathrm{P} 2 \mathrm{X} 3 \mathrm{R}$ was higher in male old rats. Representative images of immunofluorescent signals in the urinary bladder from 4'-6-diamidino-2-phenylindole (DAPI), $\beta$-actin ( $\beta$-ACT), P2X3R, and their merged signals in MYR (AD) and MOR (E-H). (I) Group analysis of the contributions of DAPI, $\beta$-actin, and P2X3R to the total signal area in bladder sections from MYR and MOR. (J), DAPI- and $\beta$-actin-normalized P2X3R signals in bladder sections from MYR and MOR. The bladders were dissected after treatment with AF-353 following CMG-EMG characterization. The scale bar in panel A indicates $50 \mu \mathrm{m}$, and applies to all images. ${ }^{\star} \mathrm{P}<0.05$ and ${ }^{* *} \mathrm{P}<0.01$ vs. the MYR group (t-test). MYR, male young rats; MOR, male old rats; CMG, cystometry; EMG, electromyography.

\section{Micturition in MYR Before and After Systemic Inhibition of P2X3R}

Simultaneous CMG/EMG demonstrated typical micturition patterns in MYR during intravesical saline infusion. Fig. 4A shows multiple micturitions where EMG was correlated with voiding events. A closer view at one of these events corroborates muscle activation (Fig. $4 \mathrm{~B}$ ) and maximal activity during IPHFO (Fig. 4C). The intramuscular application of AF-353 (10 $\mathrm{mg} / \mathrm{kg}$ ) to the same rats increased the number of micturitions in the same period of recording time (Fig. 4D), but decreased the maximal voiding pressure with a parallel reduction in EMG activity (Fig. 4E). The correlation of EMG with IPHFOs remained the same despite a decrease in IPHFO after P2X3R in- hibition. No evident NVCs were observed.

\section{Micturition in MOR Before and After Systemic Inhibition of P2X3R}

In contrast to MYR, the simultaneous EMG/CMG recordings in MOR showed significantly altered voiding patterns (Fig. 5A, B). These were characterized by a mixture of multiple NVCs (indicated with ${ }^{*}$ in Fig. 5C), SVVE, and ECs (Fig. 5C), all of them accompanied by some degree of EMG activity; these abnormal activities were observed during high intravesical pressures, but were more evident during IPHFOs (Fig. 5A-C). The systemic inhibition of P2X3R in the same MOR animals substantially affected both EMG and CMG responses. First, an in- 

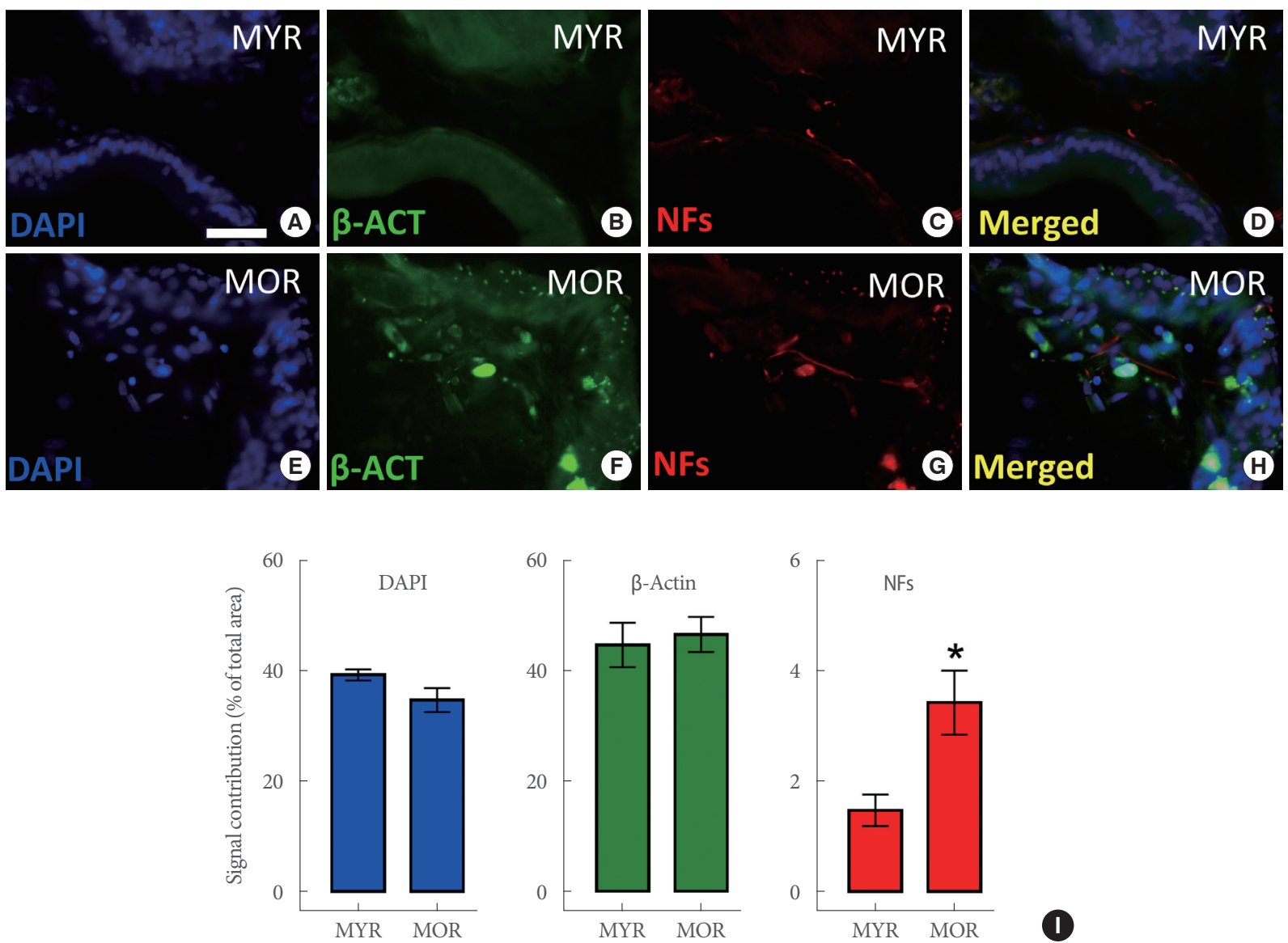

Fig. 3. Neurofilament expression was higher in male old rats. Representative images of immunofluorescent signals in the prostate from 4'-6-diamidino-2-phenylindole (DAPI), $\beta$-actin ( $\beta$-ACT), neurofilaments (NFs), and their merged signals from MYR (A-D) or MOR (E-H). (I) Group analysis of the contributions of DAPI (left), $\beta$-actin (middle), and neurofilaments (right) to the total signal area in prostate sections from MYR and MOR. Prostates were dissected after systemic treatment with AF-353 following CMG-EMG characterization. The scale bar in panel A indicates $50 \mu \mathrm{m}$, and applies to all images. ${ }^{\star} \mathrm{P}<0.05$ vs. the MYR group (t-test). MYR, male young rats; $\mathrm{MOR}$, male old rats; $\mathrm{CMG}$, cystometry; $\mathrm{EMG}$, electromyography.

crease in the intercontractile interval was observed (Fig. 5D). Second, the frequencies of NVCs and SVVE were reduced (Fig. 5E). Finally, a more typical voiding event was observed in MOR following AF-353 application (Fig. 5F).

\section{CMG and EMG Group Analysis in MYR and MOR}

A group analysis showed a higher frequency of EMG spikes in MOR than in MYR $(\mathrm{P}<0.001$ vs. MYR), and a reduction after $\mathrm{P} 2 \mathrm{X} 3 \mathrm{R}$ inhibition $(\mathrm{P}<0.01$ vs. MOR) (Fig. $6 \mathrm{~A})$. The amplitude of the spikes was also reduced in MOR $(\mathrm{P}<0.01$ vs. MYR $)$ and not altered by AF-353 application (Fig. 6B). The duration of the EMG signal was significantly higher in MOR $(\mathrm{P}<0.001$ vs. MYR) and only affected by the purinergic antagonist in MOR $(\mathrm{P}<0.05)$ (Fig. 6C). Although a trend was observed towards re- duced peak bladder pressure, no significant differences were found between MYR or MOR before or after P2X3R inhibition (Fig. 6D). The intercontractile interval was lower in MOR $(\mathrm{P}<0.05$ vs. MYR); it decreased in response to AF-353 in MYR $(\mathrm{P}<0.05)$ but increased in MOR $(\mathrm{P}<0.01)$ (Fig. 6E). The duration of IPHFOs was significantly lower in MOR than in MYR $(\mathrm{P}<0.05)$, and no effects were induced by the P2X3R antagonist in either group (Fig. 6F). Similar responses between groups were determined for voided volume (Fig. 6G). Finally, the frequency of NVCs was significantly higher in MOR $(\mathrm{P}<0.01$ vs. MYR), and decreased after injection of the P2X3R antagonist in $\operatorname{MOR}(\mathrm{P}<0.05$; Fig. $6 \mathrm{H})$. 


$$
\begin{aligned}
& \text { - Bladder pressure }\left(\mathrm{cm} \mathrm{H}_{2} \mathrm{O}\right) \\
& \text { - Rectified EMG }(\mathrm{mV}) \text { - Raw EMG (mv) }
\end{aligned}
$$
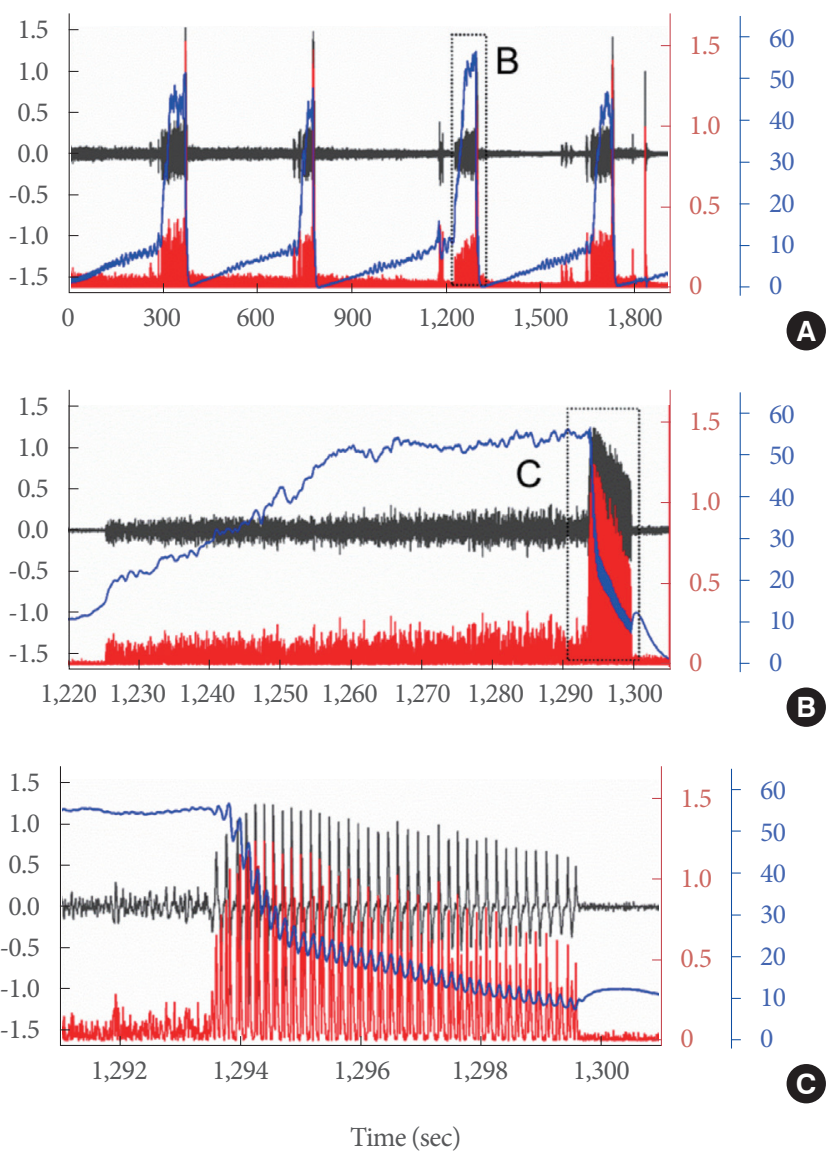

- Bladder pressure $\left(\mathrm{cm} \mathrm{H}_{2} \mathrm{O}\right)$ - Rectified EMG $(\mathrm{mV})$

- Raw EMG (mv) + AF-353 (10 mg/kg; i.m.)

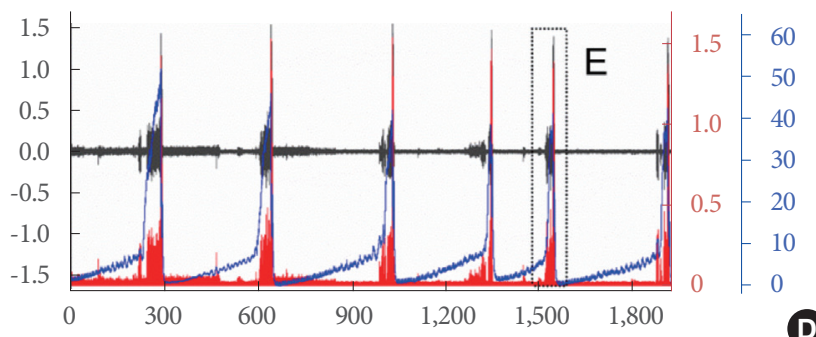

-
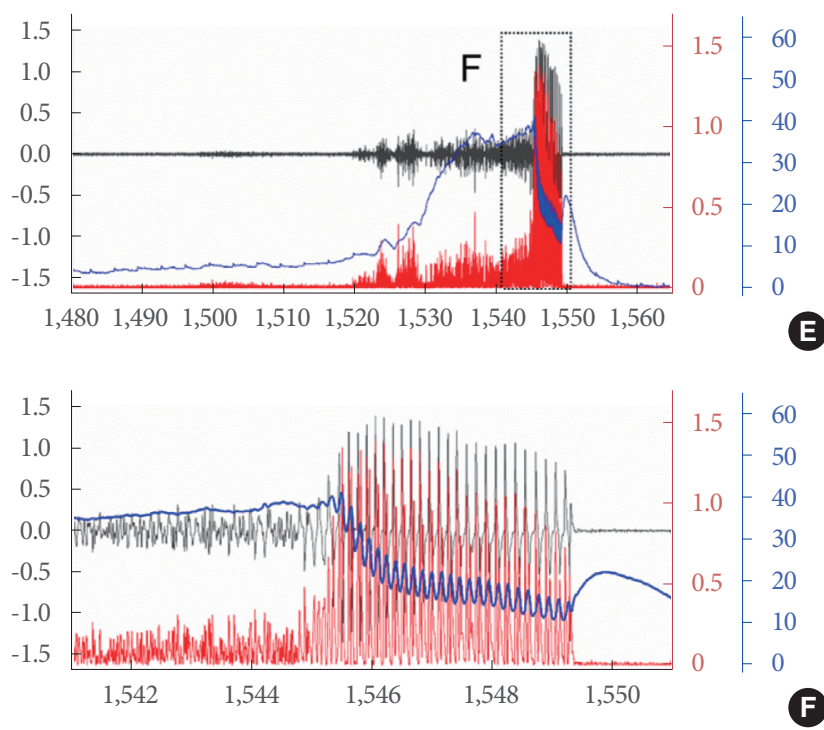

Time $(\mathrm{sec})$

Fig. 4. Systemic inhibition of $\mathrm{P} 2 \mathrm{X} 3 \mathrm{R}$ had mild effects on cystometric and electromyographic bladder responses from young male rats. (A) Representative multiple voiding events during simultaneous cystometry and bladder electromyography in control conditions. (B) Single voiding event as indicated in panel A. (C) Example of IPHFOs during the single voiding event indicated in panel B. (D) Representative pattern of the response during simultaneous cystometry and bladder electromyography after intramuscular application of 10mg/kg AF-353 in the same rat. (E) Single voiding event as indicated in panel D. (F) Characteristic IPHFOs during the single voiding event indicated in panel E after AF-353 injection. IPHFO, intraluminal pressure high-frequency oscillation; EMG, electromyography.

\section{DISCUSSION}

The present study showed a significantly higher prostate-tobody weight index in aged rats, suggesting that age-related conditions may induce a partial urethral obstruction affecting urination due to complicated voiding patterns in MOR. This observation suggest that it is possible to avoid chemically induced changes in prostate size $[16,17]$. The immunofluorescence results also revealed an increased presence of neurofilaments in MOR, supporting the possibility that pathological conditions stimulate prostatic innervation and consequently the overgrowth of the gland [18]. Additionally, the expression of P2X3R involved in the amplification of purinergic sensory signals from the bladder was also increased in the urothelium from MOR, suggesting an increased afferent tone mediated by the release of ATP [19]. As both MYR and MOR animals were treated in the same way with AF-353, we consider that this difference in the immunoreactivity of the receptor and neurofilaments did not result from exposure to the antagonist.

The current MOR model may be considered as analogous to benign prostatic hyperplasia in older humans, which may alter the normal micturition process. Prostatic hyperplasia is closely related to bladder outlet obstruction and urinary infection in males [6], although its etiology has not yet been well established 

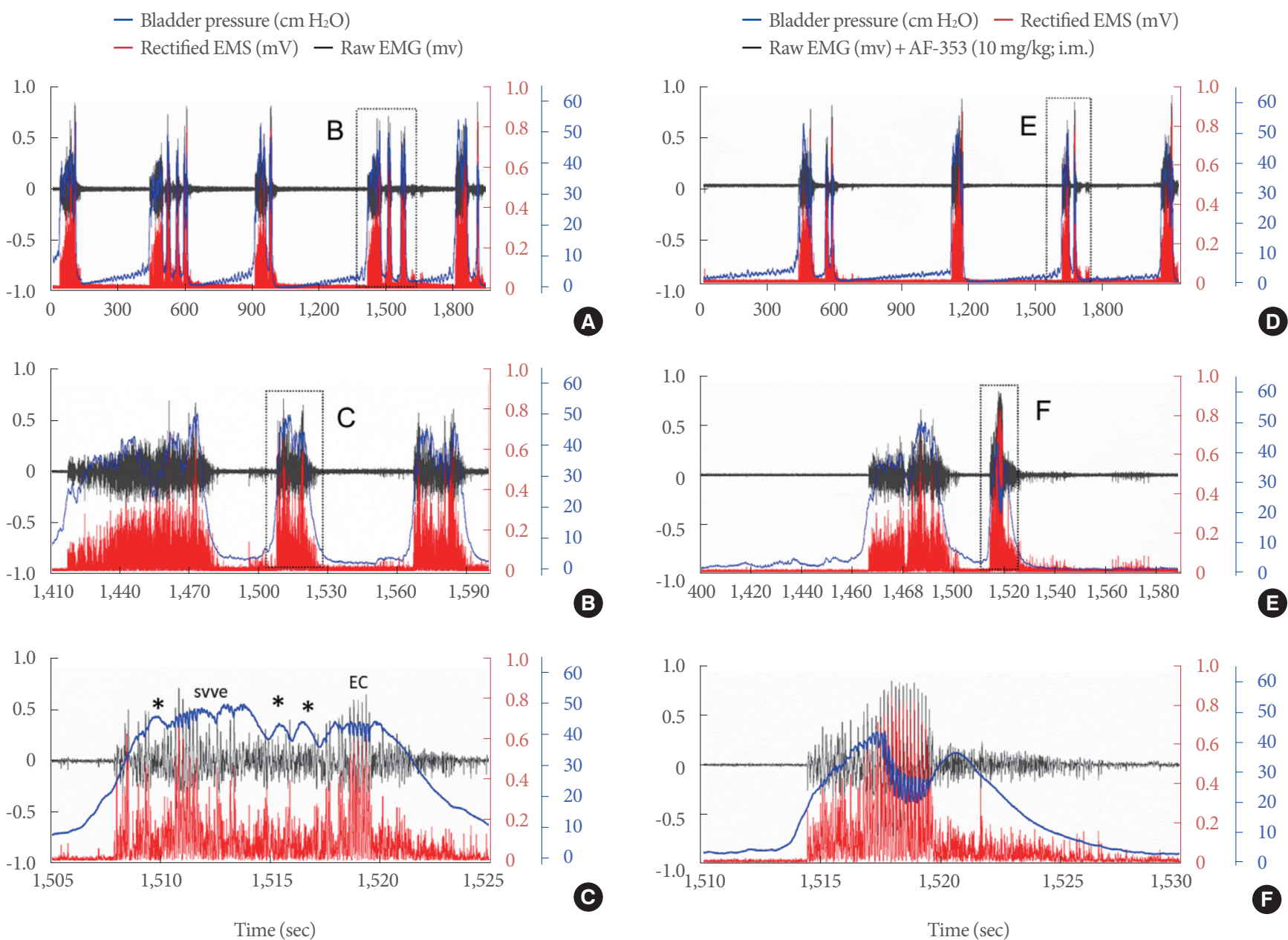

Fig. 5. Systemic inhibition of P2X3R improved cystometric and electromyographic bladder responses in male old rats. (A) Representative pattern of multiple voiding and nonvoiding events during simultaneous cystometry and bladder electromyography in control conditions. (B) Single voiding event as indicated in panel A. (C) IPHFOs during the single voiding event indicated in panel B indicating nonvoiding contractions $\left(^{*}\right)$, small volume voiding events (SVVE), and effective contractions (ECs). (D) Representative pattern response during simultaneous cystometry and bladder electromyography after intramuscular application of 10-mg/kg AF-353 to the same old animal. (E) Single voiding event as indicated in panel D. (F) IPHFOs during the single voiding event indicated in panel E. IPHFO, intraluminal pressure high-frequency oscillation; EMG, electromyography.

[20]. Although direct urethral urine flow rate was not evaluated in this study, the CMG responses suggest a dysfunctional micturition process in older animals, which may be associated with obstructive uropathy caused by a large prostate, increased prostate innervation, and enhanced afferent signals from the bladder.

The voiding patterns observed in MOR differ from those of previous murine studies in female rats under anesthesia [21], but are somewhat consistent with cystometric findings in conscious male mice [22]. This may be explained by differences in animal models and sex [6]. Nonetheless, our results suggest that a correlation between an enlarged prostate and increased ex- pression of urothelial P2X3R may explain cystometric differences observed in MOR, and that this condition may be associated with afferent hyperexcitability. Supporting this suggestion, it has been proposed that a prostate-to-bladder reflex, which is neurally mediated, may participate in regulating the micturition process [23]. Therefore, a reciprocal reflex involving the prostate and the bladder may be responsible for the impaired voiding function in MOR, and may be further related to the increased expression of P2X3R-mediated receptors in the bladder, promoting a higher expression of neurofilaments in the prostate. Our results support this hypothesis by showing the coexis- 

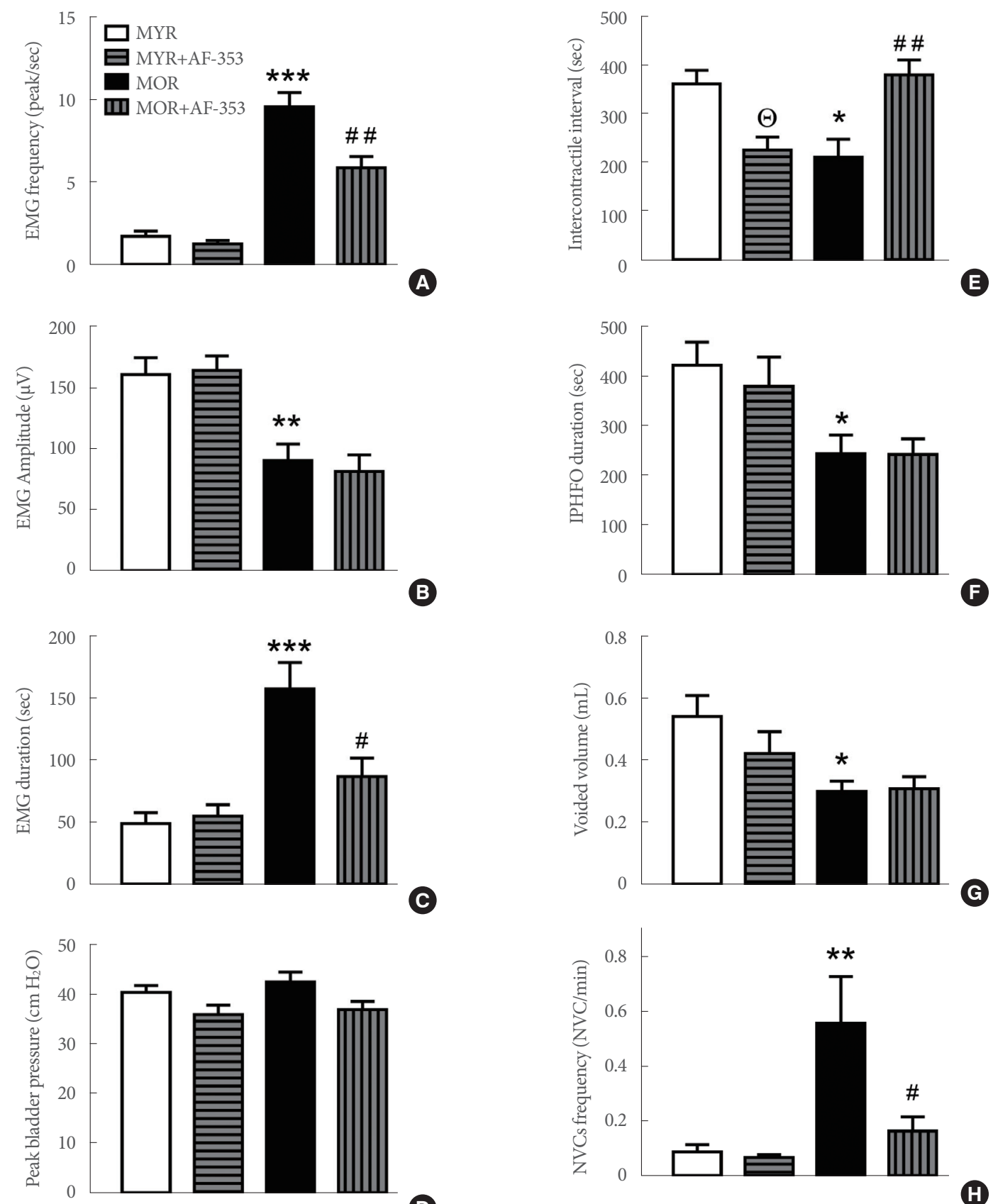

D

\section{$\boldsymbol{\Theta}$}

Fig. 6. Group analysis of electromyographic and cystometric responses in MYR and MOR before and after systemic inhibition of P2X3R. (A) EMG frequency. (B) EMG amplitude. (C) EMG duration. (D) Peak bladder pressure. (E) Intercontractile interval. (F) Intraluminal pressure high-frequency oscillations (IPHFOs). (G) Voided volume. $(\mathrm{H})$ Frequency of nonvoiding contraction (NVCs). One-way analysis of variance showed ${ }^{*} \mathrm{P}<0.05,{ }^{* *} \mathrm{P}<0.01$, and ${ }^{* *} \mathrm{P}<0.001$ between the MOR and MYR groups; ${ }^{*} \mathrm{P}<0.05$ and ${ }^{\# *} \mathrm{P}<0.01$ between MOR and MOR+AF-353; and ${ }^{\Theta} \mathrm{P}<0.05$ between MYR and MYR+AF-353. MYR, male young rats; MOR, male old rats; EMG, electromyography.

tence of prostate enlargement, increased prostate innervation, and bladder hyperactivity in MOR. Indeed, in a recent study using a formalin-induced prostatic inflammation model in rats, hyperexcitability of afferent neurons combined with bladder overactivity was observed [16], supporting the idea of more complex interactions among the prostate, the urinary bladder, 
and afferent innervation and the possibility that a localized reflex may control both organs.

In this regard, ionotropic P2X3R have been implicated as key mediators of sensory transmission in the lower urinary tract [24]. Previous reports on lower urinary tract dysfunction have found increased immunoreactivity and expression of bladder P2X3R $[12,25]$. Therefore, the observed bladder dysfunction in MOR may hypothetically also be associated with the increased expression of purinergic P2X3R in the bladder urothelium, as these receptors mainly function as mediators of bladder afferent activity following ATP release [11]. The increased urothelial expression of P2X3R in old rats may directly lead to an increased sensory tone, which is closely related to bladder overactivity. Furthermore, the current results also coincide with previous findings in male mice [12], where increased P2X3R expression, along with concurrent transmitter overflow of ATP and acetylcholine, was found in the bladder lumen. Our findings suggest that these changes in parasympathetic neurotransmitter release may also occur in MOR, and may be associated with the prostate-to-bladder reflex in a hyperexcitable mode.

As P2X3R in bladder sensory fibers and urothelial cells trigger the activation of afferent pathways [26], which may be a factor contributing to the voiding dysfunction observed in MOR, $\mathrm{P} 2 \mathrm{X} 2 / 3$ receptors were pharmacologically evaluated by systemic application of AF-353, a selective antagonist [9]. Although we did not measure nerve electrical activity, our results suggest that intramuscular injections of AF-353 in MOR resulted in marked improvements in voiding function, with significantly decreased detrusor EMG frequency and duration. Although the impact of the purinergic antagonist on EMG findings in MYR was negligible, its stronger impact on MOR can be well explained by the attenuation of enhanced purinergic signaling mediated by urothelial P2X3R, triggering the urothelial release of ATP [27] to enhance the activation of bladder afferent innervation in $\mathrm{MOR}$ animals.

These results suggest that pharmacological inhibition of P2X3R may be a promising therapeutic alternative for treating age-related bladder dysfunction. Although neither EMG nor CMG properties were restored to those observed in MYR, the overall number of nonvoiding events, indicative of bladder overactivity, improved in MOR. One reason for these differences may be that the intramuscular injections of AF-353 affected the activation of P2X3R in sensory neurons from dorsal root ganglia or spinal cord sensory neurons, making micturition effects less evident than is the case for intravenous injections or direct intravesical application, as observed in female rats with neurogenic bladder following semichronic spinal cord injury $[8,14]$. Nonetheless, it is important to keep in mind that P2X3R are also required for the activity of primary and sensory neurons involved in the micturition process [28]. Thus, the EMG/ CMG effects observed in MOR after AF-353 injection may be related to a negative effect on the activity of sensory neurons, with a concentrated effect on urothelial P2X3R at the lower urinary tract level.

In congruence with the prostate-bladder reflex, where sensory neurons in the lumbosacral spinal cord region may be responsible for their regulation [23], the application of the P2X3R antagonist could attenuate the corresponding ATP-mediated neuronal activity. For example, an intrathecal application of a similar P2X3 antagonist (AF-792) reduced the expression of phosporylated extracellular signal-related kinase in lumbosacral dorsal horn neurons from rats with bladder overactivity induced with acetic acid, resulting in improved voiding function in a bladder dysfunction model, and supporting the relevance of additional cell targets for purinergic modulation and voiding control $[29,30]$.

It is important to keep in mind that the sources for the EMG recordings from the bladder dome may include a combination of detrusor, innervation, and mechanical artifacts that may alter electrophysiological recordings. Although the source of the acquired EMG from the bladder dome remains unknown, the signals from the lower urinary tract are highly reproducible and persistently reflect voiding activity $[8,13]$. In this study, EMG responses were affected by AF-353 in MOR, which can be attributed to the fact that $\mathrm{P} 2 \mathrm{X} 3 \mathrm{R}$ play a fundamental role in the activity of sensory neurons [7], in turn attenuating the hyperexcited putative prostate-bladder reflex [23].

We took first steps towards evaluating the impact of an aging prostate on voiding dysfunction in Long-Evans rats, as a potential naturally occurring hyperplasia model. Bladder dysfunction in these aged rats may be strongly related to prostate enlargement, exacerbating a complex prostate-bladder reflex where P2X3R in urothelial cells and sensory neurons play a significant role, leading to voiding dysfunction. The results obtained after systemic application of the P2X3R antagonist AF-353 suggest that targeting these receptors can be a promising clinical alternative for treating age-related lower urinary tract symptoms. However, special care should be taken regarding the effects on other cell types expressing P2X3R, as inhibition may alter normal sensory function not related to the lower urinary tract [7]. 
A detailed comparison within the lifespan of the male rat should be performed to consolidate the current observations on micturition. Our findings bring about new perspectives for understanding voiding dysfunction and further evaluating urinary function in an animal model with potential translational impact.

\section{ACKNOWLEDGMENTS}

The authors would like to thank Betsy H. Salazar and Kristopher A. Hoffman for technical assistance.

\section{AUTHOR CONTRIBUTION STATEMENT}

- Conceptualization: $A M$

- Data curation: $C Z, A M$

- Formal analysis: $C Z, Y C, Y Z, A M$

- Funding acquisition: $T B, Y Z, A M$

- Methodology: $C Z, X L i, A M$

- Project administration: $A M$

- Visualization: T Boone, $Y C, Y Z, A M$

-Writing-original draft: $C Z, X L i, A M$

- Writing-review \& editing: $C Z, X L i, T B, Y C, Y Z, A M$

\section{REFERENCES}

1. DeWitt-Foy ME, Gill BC, Ulchaker JC. Cost comparison of benign prostatic hyperplasia treatment options. Curr Urol Rep 2019;20:45.

2. Rodriguez-Nieves JA, Macoska JA. Prostatic fibrosis, lower urinary tract symptoms, and BPH. Nat Rev Urol 2013;10:546-50.

3. Pattanaik S, Mavuduru RS, Panda A, Mathew JL, Agarwal MM, Hwang EC, et al. Phosphodiesterase inhibitors for lower urinary tract symptoms consistent with benign prostatic hyperplasia. BJU Int 2019;124:27-34.

4. Steers WD, de Groat WC. Effect of bladder outlet obstruction on micturition reflex pathways in the rat. J Urol 1988;140:864-71.

5. Kitta T, Kanno Y, Chiba H, Higuchi M, Ouchi M, Togo M, et al. Benefits and limitations of animal models in partial bladder outlet obstruction for translational research. Int J Urol 2018;25:36-44.

6. Birder LA, Kullmann AF, Chapple CR. The aging bladder insights from animal models. Asian J Urol 2018;5:135-40.

7. Fabbretti E. P2X3 receptors are transducers of sensory signals. Brain Res Bull 2019;151:119-24.

8. Salazar BH, Hoffman KA, Zhang C, Zhang Y, Cruz Y, Boone TB, et al. Modulatory effects of intravesical $\mathrm{P} 2 \mathrm{X} 2 / 3$ purinergic receptor inhibition on lower urinary tract electromyographic properties and voiding function of female rats with moderate or severe spinal cord injury. BJU Int 2019;123:538-47.

9. Gever JR, Soto R, Henningsen RA, Martin RS, Hackos DH, Panicker S, et al. AF-353, a novel, potent and orally bioavailable P2X3/ P2X2/3 receptor antagonist. Br J Pharmacol 2010;160:1387-98.

10. Takahashi R, Tyagi P, Mizoguchi S, de Groat WC, Suzuki T, Funahashi $Y$, et al. Bladder overactivity and afferent hyperexcitability induced by prostate-to-bladder cross-sensitization in rats with prostatic inflammation. J Physiol 2019;597:2063-78.

11. Takezawa K, Kondo M, Kiuchi H, Ueda N, Soda T, Fukuhara S, et al. Authentic role of ATP signaling in micturition reflex. Sci Rep 2016;6:19585.

12. Daly DM, Nocchi L, Liaskos M, Mckay NG, Chapple C, Grundy D. Age-related changes in afferent pathways and urothelial function in the male mouse bladder. J Physiol 2014;592:537-49.

13. Salazar BH, Hoffman KA, Zhang C, Kavanagh A, Zhang Y, Boone $\mathrm{TB}$, et al. Electrical activity of the bladder is attenuated by intravesical inhibition of $\mathrm{P} 2 \mathrm{X} 2 / 3$ receptors during micturition in female rats. Int Neurourol J 2017;21:259-69.

14. Munoz A, Somogyi GT, Boone TB, Ford AP, Smith CP. Modulation of bladder afferent signals in normal and spinal cord-injured rats by purinergic $\mathrm{P} 2 \mathrm{X} 3$ and $\mathrm{P} 2 \mathrm{X} 2 / 3$ receptors. BJU Int 2012;110(8 Pt B):E409-14.

15. Munoz A, Yazdi IK, Tang X, Rivera C, Taghipour N, Grossman $\mathrm{RG}$, et al. Localized inhibition of P2X7R at the spinal cord injury site improves neurogenic bladder dysfunction by decreasing urothelial P2X3R expression in rats. Life Sci 2017;171:60-7.

16. Ni J, Mizoguchi S, Bernardi K, Suzuki T, Kurobe M, Takaoka E, et al. Long-lasting bladder overactivity and bladder afferent hyperexcitability in rats with chemically-induced prostatic inflammation. Prostate 2019;79:872-9.

17. Li J, Tian Y, Guo S, Gu H, Yuan Q, Xie X. Testosterone-induced benign prostatic hyperplasia rat and dog as facile models to assess drugs targeting lower urinary tract symptoms. PLoS One 2018; 13:e0191469.

18. Ali A, Pisipati S, Tewari A. Re: Autonomic nerve development contributes to prostate cancer progression. Eur Urol 2014;65:665-6.

19. Ford AP, Undem BJ, Birder LA, Grundy D, Pijacka W, Paton JFR. P2X3 receptors and sensitization of autonomic reflexes. Auton Neurosci 2015;191:16-24.

20. Patel ND, Parsons JK. Epidemiology and etiology of benign prostatic hyperplasia and bladder outlet obstruction. Indian J Urol 2014;30:170-6.

21. Lluel P, Palea S, Barras M, Grandadam F, Heudes D, Bruneval P, et 
al. Functional and morphological modifications of the urinary bladder in aging female rats. Am J Physiol Regul Integr Comp Physiol 2000;278:R964-72.

22. Smith PP, DeAngelis A, Kuchel GA. Detrusor expulsive strength is preserved, but responsiveness to bladder filling and urinary sensitivity is diminished in the aging mouse. Am J Physiol Regul Integr Comp Physiol 2012;302:R577-86.

23. Song B, Jiang C, Wang Y, Lu Y, Li L. Newly found prostate-bladder neural reflex in rats-possible mechanism for voiding dysfunction associated with prostatitis/pelvic pain. Urology 2009;74:1365-9.

24. Andersson KE, Birder L. Current pharmacologic approaches in painful bladder research: an update. Int Neurourol J 2017;21:23542.

25. Andersson KE. Potential future pharmacological treatment of bladder dysfunction. Basic Clin Pharmacol Toxicol 2016;119 Suppl 3:75-85.
26. Vlaskovska M, Kasakov L, Rong W, Bodin P, Bardini M, Cockayne DA, et al. P2X3 knock-out mice reveal a major sensory role for urothelially released ATP. J Neurosci 2001;21:5670-7.

27. Munoz A, Gangitano DA, Smith CP, Boone TB, Somogyi GT. Removal of urothelium affects bladder contractility and release of ATP but not release of NO in rat urinary bladder. BMC Urol 2010; 10:10.

28. Ford AP, Undem BJ. The therapeutic promise of ATP antagonism at $\mathrm{P} 2 \mathrm{X} 3$ receptors in respiratory and urological disorders. Front Cell Neurosci 2013;7:267.

29. Burnstock $G$. The therapeutic potential of purinergic signalling. Biochem Pharmacol 2018;151:157-65.

30. Kaan TKY, Yip PK, Grist J, Cefalu JS, Nunn PA, Ford AP, et al. Endogenous purinergic control of bladder activity via presynaptic $\mathrm{P} 2 \mathrm{X} 3$ and P2X2/3 receptors in the spinal cord. J Neurosci 2010; 30:4503-7. 